\title{
True broad ligament fibroid mimicking ovarian mass in a postmenopausal woman
}

\author{
Lata Singh $^{1}$, Taru Gupta ${ }^{1 *}$, Snigdha Kumari², Sangeeta Gupta ${ }^{1}$
}

\begin{abstract}
${ }^{1}$ Department of Obstetrics and Gynecology, ESI PGIMSR, Basaidarapur, New Delhi, India
${ }^{2}$ Department of Obstetrics and Gynecology, PGIMER, Chandigarh, India
\end{abstract}

Received: 19 April 2019

Revised: 20 May 2019

Accepted: 30 May 2019

\author{
*Correspondence: \\ Dr. Taru Gupta, \\ E-mail: tarugupta1971@yahoo.com
}

Copyright: (c) the author(s), publisher and licensee Medip Academy. This is an open-access article distributed under the terms of the Creative Commons Attribution Non-Commercial License, which permits unrestricted non-commercial use, distribution, and reproduction in any medium, provided the original work is properly cited.

\begin{abstract}
Fibroids are benign smooth muscle tumors. Large fibroids are known to arise from the uterus, but occasionally from the broad ligament. Here, we report a case of true broad ligament fibroid which is rare and difficult to diagnose in a 57 year old postmenopausal women with a large mass of 24 weeks size gravid uterus. Patient presented with pain lower abdomen and mass in abdomen with no other co-morbidity. This broad ligament fibroid was mimicking as ovarian tumor on ultrasonography and MRI as left ovary was not visualised separately, thus leading to diagnostic dilemma of ovarian malignancy. Mass resection with total abdominal hysterectomy and bilateral salpingooopherectomy was performed. No local or distant metastasis observed, and Histopathology showed true broad ligament fibroid of left side weighing 3.57 kilograms.
\end{abstract}

Keywords: Broad ligament, MRI, Postmenopausal large fibroid, Ultrasonography

\section{INTRODUCTION}

Large fibroids arise from the uterus, but very rarely from extra-uterine sites. Broad ligament fibroid is the commonest extra-uterine site. Incidence rate of broad ligament fibroid is low $^{1}$, less than $1 \%$ and so it is both clinical and radiological difficulty in making a diagnosis. Broad ligament fibroid has been documented to reach an enormous size which can mimic ovarian malignancy. ${ }^{2}$

True broad ligament fibroid arises from tissues in the broad ligament itself, uterine vessels and ureters lie medial to the tumor. False broad ligament fibroid arises from uterus but grows laterally between the two layers of the broad ligament but retains its attachment to the uterus; uterine vessels and ureters lie lateral to the fibroid. We are reporting a case of true broad ligament fibroid because it is rare and the diagnostic difficulties in differentiating it from ovarian tumor.

\section{CASE REPORT}

We report a case of 57-year-old women, postmenopausal since 8 years, P2L2 not ligated, who presented with 6month history of lower abdominal pain.

On physical examination patient was afebrile, thin built, cachectic and haemodynamically stable. On per abdominal examination, a mass of 24 weeks size appears to be arising from the pelvis. No dilated/engorged veins and visible peristalsis. On palpation tumour was multilobulated, firm, irregular surface, non tender with slightly restricted mobility. Per speculum examination revealed cervix flushed with vagina with no abnormal discharge. 
On per vaginal examination cervix was extreme right and posterior in position. Uterus felt separately, deviated to right and bulky. An irregular mass $\sim 18-20 \mathrm{~cm}$ in size felt which firm to hard in consistency with bosselated surface. Bilateral forniceal fullness was present. Per rectal examination revealed same mass felt as in Per vaginal examination and rectal mucosa was free. Routine laboratory investigations were within normal limits. CA125 and CEA were within normal limits. Ultrasonography showed a large heteroechoic predominantly hypoechoic mass measuring $14.1 \mathrm{~cm} \mathrm{x}$ $16.6 \mathrm{~cm} \mathrm{x} 14 \mathrm{~cm}$ volume of $1097 \mathrm{cc}$ was seen arising from the pelvis and reaching upto umblicus suggestive of fibroid. Uterus was not seen separately from the mass. Right adnexa was normal but left adnexa not visualised separately.

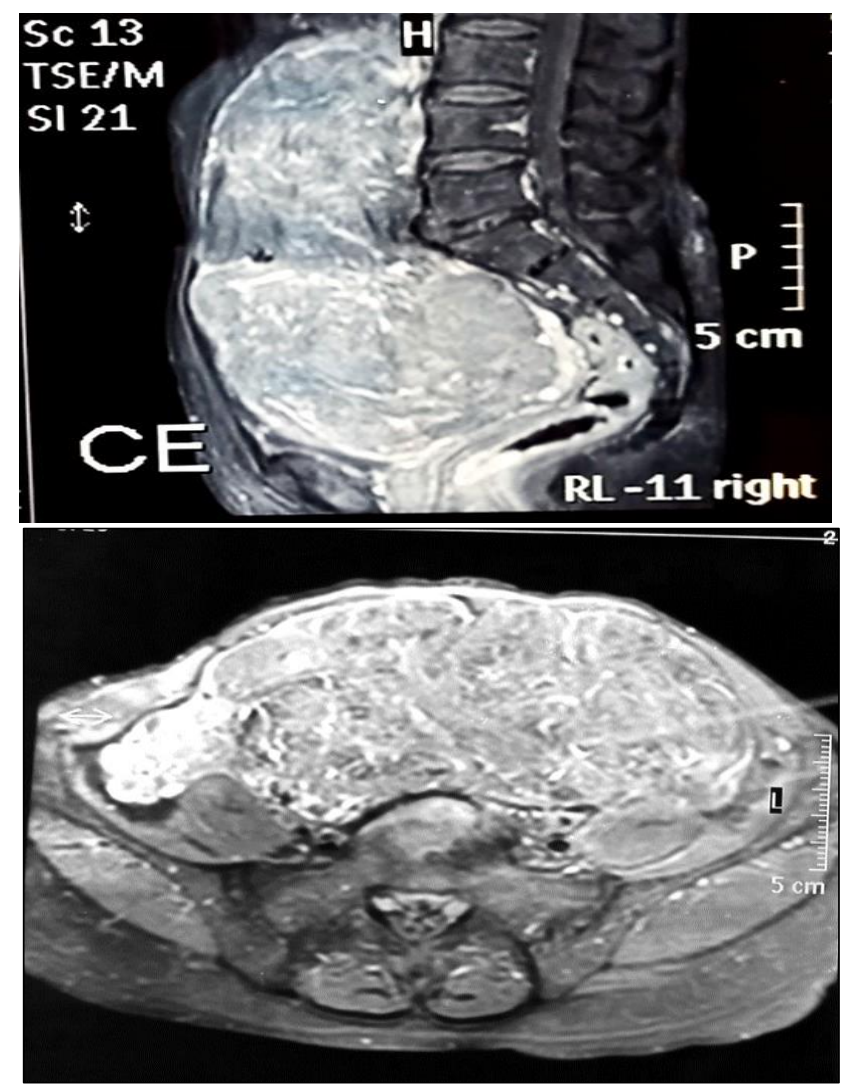

Figure 1: (A). MRI pelvis (sagittal view) showing a mild enhancing lobulated pelvic mass extending up to

L2 vertebra. (B). MRI pelvis (horizontal view) showing a mild enhancing lobulated pelvic mass extending up to $\mathrm{L} 2$ vertebra.

MRI pelvis (Figure 1A and 1B) revealed a mild enhancing lobulated mass lesion - 10.7 x $10 \times 17 \mathrm{~cm}$ in pelvis extending up to L2 vertebra displacing uterus and right ovary towards right pelvic wall, urinary bladder anteriorly and rectum posteriorly with preserved fat planes and compressing right femoral vein at S1 and IVC at L2 with bilateral hydronephrosis giving an impression of broad ligament fibroid. However, possibility of ovarian neoplasm (sex cord tumor) could not be ruled out since left ovary was not visualised. Patient was counseled about the probable diagnosis and planned for laparotomy.

\section{Intra-operative findings}

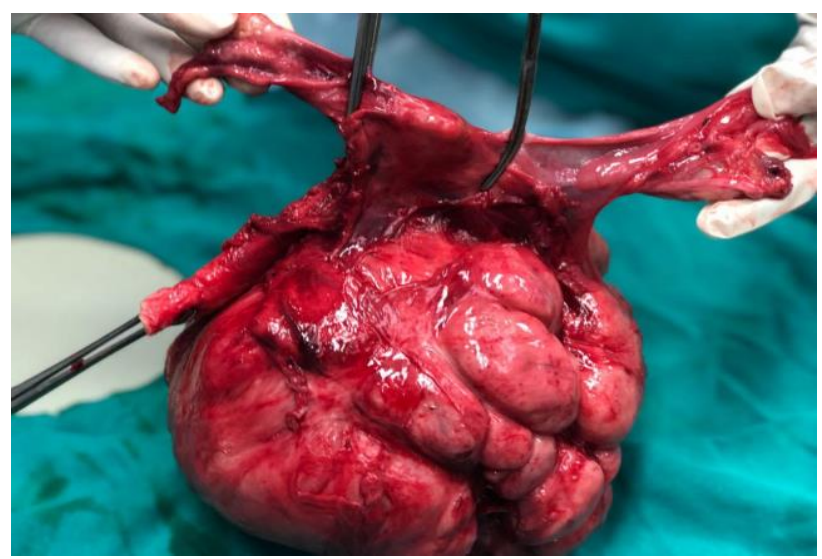

Figure 2: Uterus with tubes and ovaries along with huge true broad ligament fibroid.

A huge irregular $\sim 15 \times 17 \mathrm{~cm}$ multilobulated mass arising from left broad ligament was seen. Left round ligament and left fallopian tube was stretched out on the mass. Right round ligament and tube was normal. Bilateral ovaries were found to be normal. Uterine vessels were medial to the mass. Hence, diagnosis of true broad ligament fibroid was made. We proceeded with total abdominal hysterectomy with bilateral salpingoopherectomy.

The specimen weighed 3.57 kilograms. Cut-section of the mass showed whorled appearance suggestive of multiple fibroids (Figure 3). Post operative course was uneventful. Histopathology report was suggestive of leiomyoma.

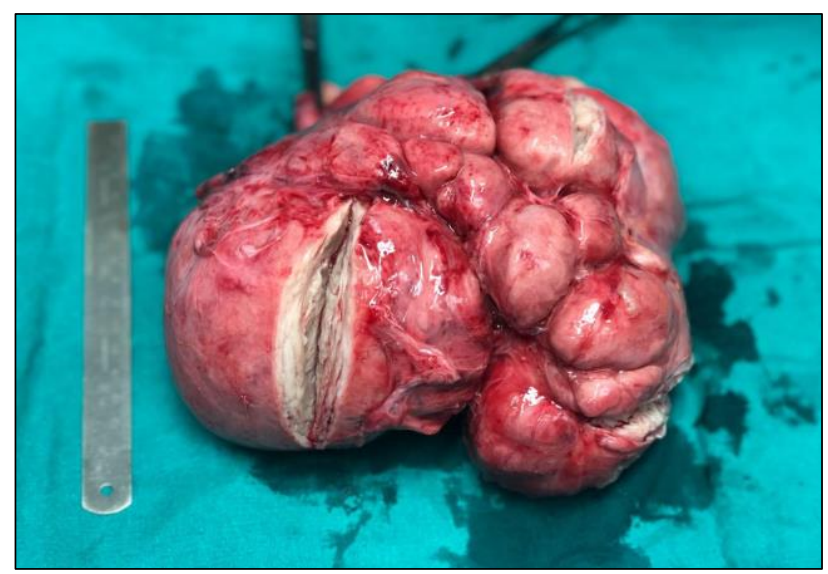

Figure 3: Cut-section of specimen showing multiple fibroids.

\section{DISCUSSION}

Uterine fibroids are the commonest benign uterine tumours found in women of reproductive age. They are 
symptomatic in $50 \%$ of cases, with the peak incidence of symptoms occurring among women in their 30 s and $40 \mathrm{~s}^{3}$ These leiomyomas are known to regress in size following menopause. However, our patient presented at 57 years of age, 6 years after menopause. Fibroids are usually diagnosed on physical examination. They can be intramural, subserosal, submucosal and cervical. Fibroids can be seen in the round, ovarian and broad ligaments. ${ }^{4}$ Among broad ligament tumours, epithelial tumors are the most common, whereas mesenchymal tumors are rare. Leiomyoma is considered most common mesenchymal tumor. ${ }^{5}$ Broad ligament fibroid can be true or false depending on the position of uterine vessels and ureter. These benign tumors are usually asymptomatic. However, they manifest clinically with various sign and symptoms if the leiomyoma reaches significant size. The location of tumors often determines the various symptoms. ${ }^{6}$ Fibroids in the broad ligament though not so common, but are well known for achieving enormous size, which may mimic a malignancy of the pelvis thereby altering the course of treatment offered. ${ }^{7-9}$ This case reports a patient who presented with pain lower abdomen and mass per abdomen that seem to be arising out of the pelvis, had features of malignancy both clinically and radiologically and exploratory laparotomy was done for the same while intra-operative findings and histopathology confirmed it as a benign broad ligament fibroid. A similar case report has been reported by Gowri et al, for its diagnostic difficulties. ${ }^{10}$ These lesions may manifest as extrauterine pelvic masses that can cause compression of urethra, bladder neck, or ureter producing symptoms of varying degrees of urinary outflow obstruction or secondary hydroureteronephrosis. Fibroid on ultrasound has a whorled appearance, with variable echogenicity depending on varying degree of degeneration, fibrosis, and calcification. ${ }^{11}$ Differential diagnosis for broad ligament fibroids is ovarian mass (both primary neoplasms and metastasis), broad ligament cyst and lymphadenopathy. Transvaginal ultrasound may be of help in diagnosing broad ligament fibroid because it allows clear visual separation of the uterus and ovaries from the mass. The distinctive MRI appearances of typical fibroids also are useful in differentiating them from solid malignant pelvic tumors but in our case left ovary was not visible on ultrasound and MRI so it was difficult to rule out possibility of ovarian malignancy.

Funding: No funding sources Conflict of interest: None declared

Ethical approval: Not required

\section{REFERENCES}

1. Kumar P, Malhotra N. Jeffcoate's Principles of Gynaecology. $7^{\text {th }}$ Ed. New Delhi: Jaypee Brothers. Tumors of the corpus uteri. 2008:492.

2. Dayananda KR, Vaibhav P, Sujid J, Sujatha ND. Broad Ligament Fibroid Mimicking as Ovarian Tumour on Ultrasonography and CT Scan. J Clin Imaging Sci. 2013:3,8.

3. Wise LA, Palmer JR, Stewart EA, Rosenberg L. Age-specific incidence rates for self-reported uterine leiomyomata in the black women's health study. Obstet Gynecol. 2005;105(3):563-8.

4. Jonathan S Berek. Benign Diseases of the Female Reproductive Tract. In: Novak's Gynecology $13^{\text {th }}$ Ed. Lippincott Williams \& Wilkins, Philadelphia; 2002:380.

5. Thor AD, Young RH, Clement PB. Pathology of fallopian tube, broad ligament, peritoneum and pelvic soft tissues. Hum Pathol. 1991;22(9):856-67.

6. Stewart EA. Uterine Fibroids. Lancet. 2001;357(9252):293-8.

7. Malik R, Agarwal G. A Large Cystic Degenerating Broad ligament Leiomyoma Masquerading as Ovarian Malignancy. JCR. 2015;5(2):486-9.

8. Mallick D, Saha M, Chakrabarti S, Chakraborty J. Leiomyoma of broad ligament mimicking ovarian malignancy- report of a unique case. Kathmandu Univ Med J (KUMJ). 2014;12(3):219-21.

9. Yadav S, Maheswari B, Sagar N, Mallya V, Khurana N, Gupta S. Broad Ligament Lipoleiomyoma Masses: Two curious cases masquerading as ovarian carcinomas. Sultan Qaboos Univ Med J. 2018;17(4):e477-e80.

10. Gowri V, Sudheendra R, Oumachigui A, Sankaran V. Giant broad ligament leiomyoma. Int J Gynaecol Obstet 1992;37(3):207-10.

11. Fasih N, Prasad Shanbhogue AK, Macdonald DB, Fraser-Hill MA, Papadatos D, Kielar AZ, et al. Leiomyomas beyond the uterus: Unusual locations, rare manifestations. Radiographics. 2008;28(7):1931-48.

Cite this article as: Singh L, Gupta T, Kumari S, Gupta S. True broad ligament fibroid mimicking ovarian mass in a postmenopausal woman. Int $\mathbf{J}$ Reprod Contracept Obstet Gynecol 2019;8:2910-2. 